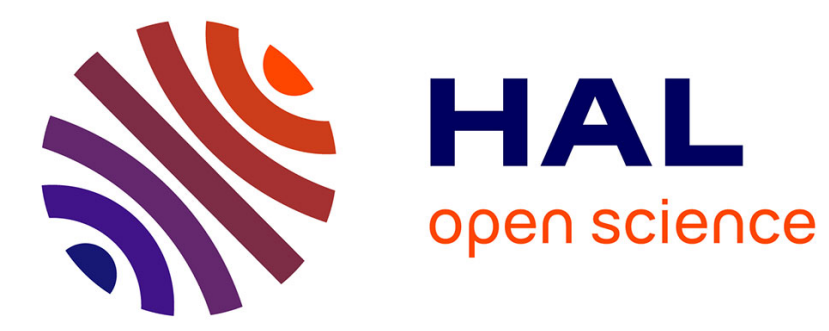

\title{
Rôle du tempérament et de la conscience émotionnelle dans la prise de risque chez des adolescents
}

Vincent Bréjard, Agnès Bonnet, Jean-Louis Pedinielli

\section{To cite this version:}

Vincent Bréjard, Agnès Bonnet, Jean-Louis Pedinielli. Rôle du tempérament et de la conscience émotionnelle dans la prise de risque chez des adolescents. L'Encéphale, 2012, 38 (1), pp.1-9. 10.1016/j.encep.2011.04.005 . hal-01314716

\section{HAL Id: hal-01314716 https://hal-amu.archives-ouvertes.fr/hal-01314716}

Submitted on 22 Sep 2016

HAL is a multi-disciplinary open access archive for the deposit and dissemination of scientific research documents, whether they are published or not. The documents may come from teaching and research institutions in France or abroad, or from public or private research centers.
L'archive ouverte pluridisciplinaire HAL, est destinée au dépôt et à la diffusion de documents scientifiques de niveau recherche, publiés ou non, émanant des établissements d'enseignement et de recherche français ou étrangers, des laboratoires publics ou privés. 


\title{
Rôle du tempérament et de la conscience émotionnelle dans la prise de risque chez des adolescents
}

\author{
Vincent Bréjard, Agnès Bonnet, Jean-Louis Pedinielli
}

\section{Introduction}

L'adolescence est une période critique du développement psychologique où apparaissent des conduites d'expérimentation, de transgressions diverses. Mais pour une minorité non négligeable d'adolescents, la répétition, la fréquence et l'intensité des comportements peuvent amener à les considérer non plus comme entrant dans le cadre d'un processus développemental, mais comme des conduites à risques. Elles suscitent l'intérêt du clinicien par les conséquences potentiellement négatives en termes traumatologiques, psychosociaux et psychopathologiques qu'elles comportent. Elles constituent un phénomène complexe influencé par de multiples facteurs: psychologiques, sociaux, environnementaux $[43,47]$. Parmi ceux-ci, l'importance de certaines dimensions tempéramentales telles que la recherche de sensations et la recherche de nouveauté, et de déficits émotionnels tels qu'anhédonie ou alexithymie ont été soulignés.

\section{Adolescence, conduites à risques et tempérament}

Il n'existe pas de définition univoque du tempérament. Ce concept se définit comme un ensemble de différences individuelles sous-tendues par des bases neurobiologiques, voire génétiques [48]. Ces différences individuelles s'expriment par des modalités spécifiques de réactivité émotionnelle et comportementale, en particulier aux stimuli de l'environnement. Deux dimensions spécifiques semblent plus particulièrement impliquées dans la prise de risque répétée: la recherche de stimulations fortes et variées, et l'impulsivité - désinhibition, par ailleurs. Deux modèles ont été plus particulièrement utilisés pour conceptualiser ces dimensions: celui de la recherche de nouveauté de Cloninger, et celui de la recherche de sensation de Zuckerman.

La recherche de sensation se définit comme la tendance à éviter ou rechercher des stimulations intenses. Dans sa formulation initiale, la recherche de sensation était conçue comme déterminée par le niveau d'activation corticale (faible ou élevé) du sujet. Des critiques ont amené Zuckerman à étendre son modèle à cinq facteurs dans une perspective plus biopsychologique, puis à une complexification de la dimension de recherche de sensation en recherche impulsive et non socialisée de sensations [8]. Différentes études ont révélé des relations significatives et positives entre recherche de sensations et prise de risques dans le domaine sexuel [52], dans celui des sports extrêmes $[3,25,39]$, ou dans la consommation [15,22] et l'abus de substances psychoactives [24,55]. Dans une étude française chez l'adolescent [38], une relation forte entre recherche de sensations et consommation de substances a été retrouvée, particulièrement chez les adolescents présentant une symptomatologie dépressive. L'auteur explique ces résultats en émettant l'hypothèse que la recherche de sensations fortes pourrait constituer une tentative d'adaptation à un déficit émotionnel lié à l'état dépressif.

La recherche de nouveauté s'inscrit quant à elle dans un modèle du tempérament d'emblée psychobiologique proposé par Cloninger et al. [9], visant à une application tant en population clinque que générale. Ce modèle à l'origine tridimensionnel (recherche de nouveauté, évitement du danger, dépendance à la récompense) a été modifié par 
l'isolement d'une sous-dimension de la dépendance à la récompense, renommée persistance (et ce pour des raisons psychométriques et conceptuelles) puis complété ultérieurement par l'adjonction de trois dimensions du caractère (acquises au cours du développement) permettant de préciser le degré de maturité de la personnalité et donc la présence ou l'absence de trouble de la personnalité. La recherche de nouveauté se définit comme la tendance à répondre par l'excitation ou l'exaltation à des stimuli nouveaux, et serait reliée à l'activation comportementale et au système dopaminergique. L'émotion de base qui lui serait associée serait la colère. Cette dimension a fait l'objet de nombreux travaux dans le domaine des addictions et des troubles du comportement. Cloninger et al. ont ainsi montré qu'une forte recherche de nouveauté, un faible évitement du danger et une faible dépendance à la récompense interviennent dans l'addiction, en particulier à l'alcool [10-12]. Ce modèle apparaît prédicteur de l'alcoolisme à l'âge adulte. D'autres études ont appliqué ce modèle à différents types d'abus de substances psychoactives $[1,54]$. Chez l'adolescent, il a également été utilisé, mais avec des résultats contradictoires: Masse et Tremblay [37] ne retrouvent en effet pas de relation entre recherche de nou veauté et consommation de substances. À l'inverse Wills et al. observent une relation significative entre recherche de nouveauté et consommation de substances, associée à divers autres comportements «à problèmes» $[56,57]$.

Il semblerait que la recherche de sensations soit en relation avec des prises de risques de type physique comme les sports extrêmes, alors que la recherche de nouveauté serait plutôt reliée à la recherche de stimulation et à l'activation comportementale, impliquant des tendances antisociales [58]. Les deux dimensions se recouvriraient donc partiellement mais ne rendraient pas forcément compte de la même expression comportementale.

Toutefois, la recherche de sensations et la recherche de nouveautés, pourraient constituer les modalités d'expression d'un soubassement général et motivationnel commun, constitué d'une tendance à rechercher activement des stimulations, associé à une activation comportementale exploratoire. Il faut souligner que selon Cloninger et Zuckerman, les dimensions de leur modèle en relation avec les addictions seraient sous-tendues par le fonctionnement dopaminergique, lui-même considéré comme impliqué dans le fonctionnement addictif [53].

\section{Psychopathologie des émotions et conduites à risques}

Différentes études ont analysé les relations entre psychopathologie de l'émotion et conduites à risques. Si l'on repère classiquement comme faisant partie intégrante des troubles émotionnels des entités psychopathologiques telles que les troubles anxieux, les troubles de l'humeur, les dysfonctionnements émotionnels dans la schizophrénie [49], l'alexithymie a été la première définition d'un concept spécifiquement relié à un trouble du fonctionnement cognitivo-émotionnel dans une approche biopsychosociale. Dans le champ des conduites pathologiques, telles que les addictions, l'alexithymie a fait l'objet d'une importante somme de contributions, notamment françaises [19,35,51].
Dans la dépendance alcoolique, un fonctionnement alexithymique est retrouvé chez une proportion significative de patients, mais associée à la dépression, ce qui pose le problème de l'indépendance entre ces deux concepts [19]. Cette association entre alexithymie et dépression est également retrouvée dans la dépendance au tabac [26], aux substances psychoactives hors alcool $[34,52]$ ainsi que dans le jeu pathologique $[36,46]$. Une explication possible du fonctionnement alexithymique a été proposée dans une perspective développementale par Lane et Schwartz dans le cadre de travaux issus du champ des neurosciences, en s'appuyant sur une intégration des théories de Piaget et de Werner $[29,30]$. Elle considère que le développement émotionnel suit une ligne développementale hiérarchique, caractérisée par une succession de niveaux de «conscience émotionnelle». La conscience émotionnelle décrit la capacité d'un individu à faire l'expérience d'états subjectifs différenciés, à les interpréter correctement, ainsi qu'à les identifier et les imaginer chez autrui. Elle serait un type de traitement cognitif qui correspondrait à cinq niveaux de transformation structurale tout au long du développement:

- la prise de conscience de sensations corporelles (avec le développement des premières capacités de symbolisation):

- la conscience des sensations dans l'action motrice (l'enfant prend conscience qu'il peut s'autostimuler) et de la possibilité de l'existence de sensations chez autrui ;

- la différenciation progressive d'émotions de base, proches des sensations perçues;

- l'apparition de la capacité à discriminer des émotions complexes;

- la capacité à ressentir, décrire chez soi-même et chez autrui des combinaisons d'émotions complexes et différenciées.

Ces cinq niveaux de conscience des émotions peuvent être considérés comme les équivalents émotionnels des stades de développement de la pensée. En effet, expérimenter des états émotionnels complexes suppose d'avoir développé des aptitudes quant au traitement et à l'intégration des informations perceptivo-sensorielles concernant ses propres états internes. Cela implique la capacité à construire des représentations de plus en plus complexes permettant de discriminer et de ressentir un large panel d'expériences émotionnelles. Afin d'évaluer le niveau de conscience émotionnelle, une échelle a été développée, l'échelle de niveaux de conscience émotionnelle (Level of Emotionnal Awareness Scale ou LEAS), qui a été traduite et validée en français [7].

Pour Lane et al. [31], les caractéristiques de certaines phases du développement cognitivo-émotionnel peuvent être comparées au fonctionnement émotionnel pathologique de certains patients, notamment dans le cas de l'alexithymie. Pour ces auteurs les déficits émotionnels rencontrés pourraient correspondre à des arrêts développementaux à des stades précoces du développement émotionnel. Un faible niveau de conscience émotionnelle a été retrouvé dans différentes entités psychopathologiques tels que les troubles unipolaires et les troubles anxieux $[16,45]$, les troubles des conduites alimentaires $[6,17]$, ainsi 
que la dépendance à l'alcool [2] ou la polytoxicomanie [23]. Chez l'enfant et l'adolescent, un faible niveau de conscience émotionnelle est retrouvé associé aux troubles internalisés [48] et de faibles compétences dans les capacités de traitement de l'information émotionnelle ont été observées chez les adolescents ayant le plus tendance à prendre des risques [21].

Dans une perspective s'inspirant des propositions de Solange Carton (qui se réfère pour sa part au modèle de Zuckerman), nous pensons que la recherche de stimulations et l'activation comportementale dans ses aspects pathologiques peut être mis en perspective de certaines «modalités de traitement de l'émotion» dont le déficit de précision et de complexité implique la conscience qu'un sujet a de ses états émotionnels [8]. En ce sens, l'effet du tempérament sur les conduites à risques peut être lié à des dimensions cognitivo-émotionnelles influencées par les interactions avec l'environnement, ce qui justifie l'étude de leur articulation dans les conduites où la recherche de stimulations est au premier plan.

Afin de tenter de rendre compte de facteurs potentiels de vulnérabilité chez les adolescents à s'engager dans des conduites à risques, nous avions deux objectifs majeurs dans cette étude. Le premier était de confirmer les travaux précédents portant sur les relations entre dimensions du tempérament et conduites à risques, le second était d'analyser les contributions respectives du tempérament et de la conscience émotionnelle à la propension à s'engager dans des conduites à risques.

\section{Méthode}

\section{Participants}

Notre échantillon a été constitué à partir d'une population d'adolescents scolarisés dans différents établissements (collèges d'enseignement général, lycées professionnels, lycées d'enseignement généraux) des départements des Bouches-du-Rhône (13) et de la Vienne (86). L'inclusion dans l'étude s'est réalisée de septembre 2003 à juin 2004. Les adolescents ont été rencontrés par l'expérimentateur en classe et il leur a été expliqué les objectifs de l'étude. Leur accord écrit, ainsi que celui de leurs parents et du chef d'établissement a été demandé, après explication des objectifs de l'étude. Au final, sur 565 adolescents contactés, 34 ont refusé de participer à l'étude et 488 ont retourné des questionnaires exploitables soit $86,37 \%$ (âge moyen $=14,93 \pm 1,44$ ). Ainsi 257 garçons (âge moyen $=15,38 \pm 1,51$ ) et 231 filles (âge moyen $=14,52 \pm 1,23$ ) ont participé à l'étude.

Les passations se déroulaient en classe en notre présence ou celle de deux collaborateurs. Les élèves ayant participé à la recherche sont issus de classes de cinquième à troisième de collège, de filières quatrième et troisième SEGPA et de deuxième à terminale de lycées d'enseignement général et de lycées professionnels.

\section{Outils}

L'évaluation des conduites à risques a été réalisée par la Youth Risk Behavior Surveillance Scale dans sa forme abrégée [18]. L'échelle d'évaluation des risques chez les jeunes est dans sa version originale une échelle d'autoévaluation en dix items constitués d'affirmations auxquelles le sujet doit répondre par un choix forcé de type vrai/faux. Il n'existe à ce jour pas de traduction française de cet instrument ce qui nous a donc amené à effectuer la traduction en double-sens: anglais-français et françaisanglais. La consistance interne satisfaisante de l'instrument pour cette étude est correcte (alpha de Cronbach $=0,71$ ).

Nous avons également adapté une forme "enseignante» qui nous a permis de disposer d'une double évaluation des conduites à risques chez les adolescents. Nous avons croisé les deux évaluations afin de n'inclure dans l'échantillon que les adolescents dont l'autoévaluation recoupait l'hétéroévaluation, mais n'avons conservé que les autoévaluations pour les traitements statistiques.

Le questionnaire tridimensionnel de la personnalité a été utilisé pour évaluer le tempérament. Il s'agit d'un inventaire de 100 items permettant d'évaluer les trois dimensions du tempérament que sont la recherche de nouveauté (RDN), l'évitement du danger (ED) et la dépendance a la récompense (DR), la persistance ( $P$ ), conformément au modèle de Cloninger et al. [13]. Il a fait l'objet de plusieurs études de validation en français confirmant ses propriétés psychométriques [32,33]. Le TPQ a été utilisé à plusieurs reprises avec des échantillons d'adolescents, notamment dans le cadre de recherches sur les conduites à risques et la dépression $[56,57]$.

La LEAS ou échelle de niveau de conscience émotionnelle a permis d'évaluer la conscience émotionnelle. Cet instrument d'évaluation consiste en 20 items dans lesquels on demande au sujet de s'imaginer une courte scène le mettant en interaction avec une autre personne. Puis le sujet doit répondre à deux questions : l'une porte sur ce qu'il ressentirait dans la situation, l'autre porte sur ce que l'autre personne ressentirait, en commençant la phrase par «je me sentirais ou je ressentirai...» et par «l'autre personne ressentirait ou se sentirait...». La cotation des réponses s'effectue en repérant dans le texte des réponses les mots représentant des émotions et grâce à une grille de cotation, qui propose une liste de termes émotionnels et une note s'y référant. Les réponses sont cotées de 0 à 5 et permettent d'obtenir un score sur une échelle de 0 à 100. La LEAS comporte deux sous-échelles permettant d'obtenir une évaluation des capacités du sujet à être conscient de ses propres états émotionnels, ainsi que de ses capacités à les conceptualiser chez autrui. La structure factorielle de l'échelle ainsi que ses qualités psychométriques sont satisfaisantes $[7,28]$. Cependant, aucune étude de validation n'étant disponible chez l'adolescent, nous avons été amenés à évaluer la possibilité d'utiliser la LEAS avec cette population dans le cadre d'un prétest. Celui-ci nous a permis d'observer que la formulation des items n'était pas totalement adaptée à cette tranche d'âge. La première remarque qui a été faite par les adolescents lors du prétest était que l'utilisation de la forme «vous» posait problème, et qu'ils préféraient l'utilisation du tutoiement. Par ailleurs, un certain nombre de situations impliquant une référence au monde du travail (patron, supérieur, projet à réaliser), étaient considérées comme hors contexte. Nous les avons donc modifiées par des situations issues du cadre scolaire, en remplaçant par exemple «votre patron. .. p par «votre professeur...» [4]. 
Dans cette forme modifiée, la consistance interne (a de Cronbach $=.83$ ) est congruente avec celle obtenue dans la forme initiale [28].

Nous avons réalisé conformément aux recommandations des auteurs une double cotation sur $25 \%$ du protocole. La corrélation entre les deux séries de cotation était de 0,92 , similaire aux travaux francophones déjà publiés chez l'adulte.

\section{Analyses statistiques}

Nous avons effectué les analyses statistiques en deux étapes. Une première phase a consisté en des analyses descriptives sur les différentes variables. Pour cela, nous avons utilisé des méthodes classiques de comparaison de moyennes (test $t$ de Student). Une seconde phase a consisté en des analyses multivariées afin d'évaluer les relations entre les variables dépendantes et indépendantes. Nous avons pour cela utilisé les procédures suivantes: corrélations ( $r$ de Bravais Pearson), régressions linéaires multiples. Les analyses ont été effectuées avec le logiciel d'analyse statistique de données SPSS 11 (SPSS Inc.).

\section{Résultats}

Les statistiques descriptives ainsi que l'analyse des différences intersexes ( $t$ de Student) sont présentées dans l $\epsilon$ Tableau 1. Les garçons apparaissent en moyenne significativement plus âgés que les filles, mais avec une différence peu importante (moins d'un an). Par ailleurs, ils présentent un score significativement plus élevé de conduites à risques. Concernant les dimensions de tempérament, il n'existe pas de différence significative à la recherche de nouveauté, ni à celle de dépendance à la récompense et de la persistance. En revanche, les filles présentent un niveau plus élevé d'évitement du danger $(t=-2,479$; $p<0,05)$. Dans le même sens, elles présentent un niveau de conscience émotionnelle global supérieur aux adolescents $(t=-3,509 ; p<0,0001)$, qui est retrouvé pour les deux sous-dimensions du NCE soi $(t=-3,275 ; p=0,001)$ et autre personne $(t=-3,455 ; p<0,0001)$. Enfin, si l'on se base sur la médiane de l'échantillon à la variable conduite à risques (med $=2$ ), on remarque que $22 \%$ des adolescents présentent un niveau significatif de conduite à risques, tandis que $78 \%$ n'en présentent pas.

Les analyses de corrélations entre les variables montrent plusieurs liaisons significatives, allant de faibles $(0,10<r<0,30)$ à modérées $(0,30<r<0,50)$ [14]. Les résultats sont présentés dans le Tableau 2 , et ne seront repris que ceux correspondant aux relations entre variables explicatives (tempérament et niveau de conscience émotionnelle) et variable à expliquer (conduites à risques). Concernant les dimensions du tempérament: il existe une corrélation modérée entre recherche de nouveauté et conduites à risques $(r=0,43 ; p<0,01)$, ainsi qu'une relation négative et faible entre évitement du danger et conduites à risques $(r=-0,26 ; p<0,01)$. Le niveau de conscience émotionnel général entretient quant à lui une relation négative et modérée avec les conduites à risques $(r=-0,45 ; p<0,01)$, de même que les deux sous-dimensions soi $(r=-0,47 ; p<0,01)$ et autre personne $(r=-0,37 ; p<0,01)$.

Afin de préciser les relations entre tempérament, conscience émotionnelle et conduites à risques, nous avons réalisé une première analyse en régression multiple sur l'ensemble de l'échantillon, avec comme variables contrôlées l'âge et le sexe (codés de manière binaire), et comme critères dans le modèle les quatre dimensions du tempérament et les deux dimensions du niveau de conscience émotionnelle. Les résultats sont présentés dans le Tableau 3. Le modèle global contribue à $33 \%$ de la variance des conduites à risques $\left(R^{2}=0,33 ; F=30,78 ; p<0,0001\right)$. Trois dimensions ont un effet significatif: la recherche de nouveauté $(p<0,0001)$, et le niveau de conscience émotionnelle soi $(p<0,0001)$ et autre personne $(p=0,0001)$. Une seconde série de régressions a été réalisée afin d'évaluer la contribution potentielle des dimensions du tempérament à la conscience émotionnelle: nous avons constaté

Tableau 1 Statistiques descriptives et différences intersexes aux variables âges, tempérament, et niveau de conscience émotionnelle.

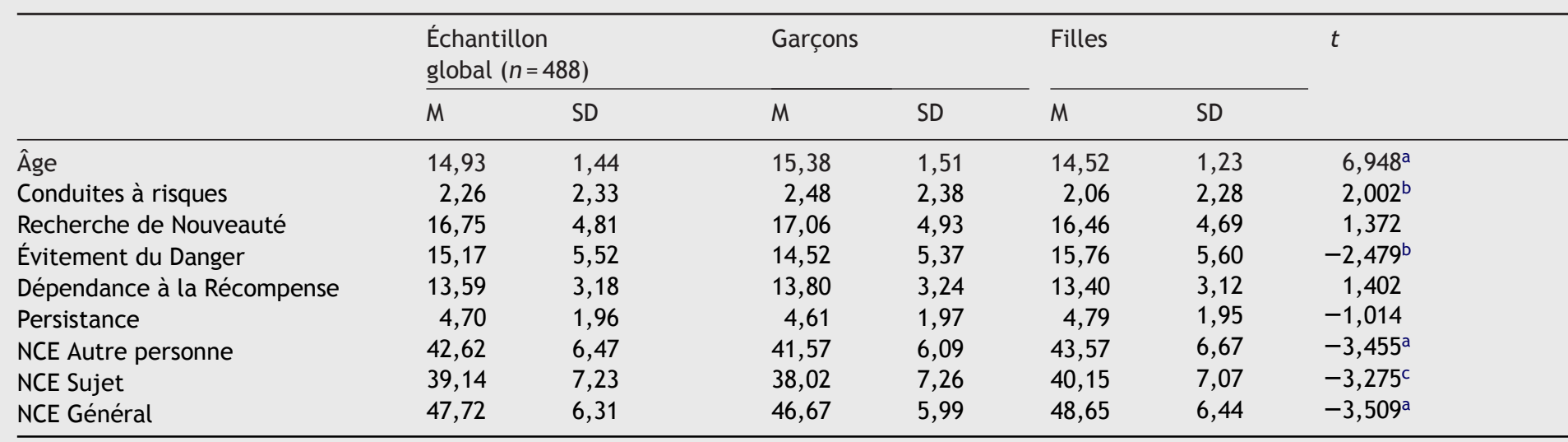

NCE : niveau de conscience émotionnelle.

$$
\begin{aligned}
& \text { a } p<0,0001 . \\
& \text { b } p<0,05 . \\
& \text { c } p=0,001 .
\end{aligned}
$$


Tableau 2 Corrélations ( $r$ de Bravais-Pearson) entre l’âge, les conduites à risque, la tempérament et le niveau de conscience émotionnelle.

\begin{tabular}{|c|c|c|c|c|c|c|c|c|c|c|}
\hline & & 1 & 2 & 3 & 4 & 5 & 6 & 7 & 8 & 9 \\
\hline 1 & Âge & 1,00 & $0,18^{a}$ & $0,15^{\mathrm{a}}$ & $-0,19^{a}$ & 0,10 & $-0,07$ & $-0,13^{a}$ & $-0,18^{a}$ & $-0,15^{a}$ \\
\hline 2 & Conduites à risques & & 1,00 & $0,43^{a}$ & $-0,26^{a}$ & $0,11^{\mathrm{b}}$ & $-0,17^{a}$ & $-0,37^{a}$ & $-0,47^{a}$ & $-0,45^{a}$ \\
\hline 3 & Recherche de Nouveauté & & & 1,00 & $-0,45^{\mathrm{a}}$ & 0,05 & $-0,35^{a}$ & $-0,10^{b}$ & $-0,30^{a}$ & $-0,13^{a}$ \\
\hline 4 & Évitement du Danger & & & & 1,00 & $-0,07$ & $0,20^{\mathrm{a}}$ & $0,14^{\mathrm{a}}$ & $0,29^{a}$ & $0,15^{a}$ \\
\hline 5 & Dépendance à la Récompense & & & & & 1,00 & 0,03 & $-0,03$ & $-0,08$ & $-0,07$ \\
\hline 6 & Persistance & & & & & & 1,00 & $-0,01$ & $0,12^{\mathrm{a}}$ & 0,03 \\
\hline 7 & NCE Autre personne & & & & & & & 1,00 & $0,62^{\mathrm{a}}$ & $0,88^{\mathrm{a}}$ \\
\hline 8 & NCE Sujet & & & & & & & & & $0,73^{a}$ \\
\hline 9 & NCE Général & & & & & & & & & 1,00 \\
\hline
\end{tabular}

NCE : niveau de conscience émotionnelle.

${ }^{\mathrm{a}} p<0,01$.

b $p<0,05$.

que la recherche de nouveauté et l'évitement du danger expliquent $12 \%$ de la variance $\left(R^{2}=0,12 ; F=16,71\right.$; $p<0,0001)$ de la conscience émotionnelle soi, alors que le pourcentage de variance de la conscience émotionnelle autre personne expliquée par ce même modèle est négligeable bien que significative $\left(R^{2}=0,02 ; F=3,02 ; p=0,02\right)$.

\section{Discussion}

Cette recherche avait comme objectif d'analyser les relations entre deux dimensions psychologiques - le tempérament et le niveau de conscience émotionnelle - et l'engagement dans des conduites à risques chez les adolescents. Elle constituait à notre connaissance le premier travail sur ce thème et cette population. Pour cela nous avons réalisé dans un premier temps des analyses corrélationnelles afin de confirmer l'existence de ces relations, puis dans un second temps nous avons évalué la contribution relative du tempérament et de la conscience émotionnelle par un modèle permettant de préciser quelles dimensions spécifiques étaient susceptibles de constituer des facteurs de vulnérabilité à s'engager dans des conduites à risques chez les adolescents. Les résultats obtenus confirment partiellement nos hypothèses concernant le rôle respectif de deux dimensions du tempérament et du niveau de conscience émotionnelle. La recherche de nouveauté apparaît être le principal facteur de vulnérabilité à l'engagement dans des prises de risques. Cela confirme les travaux précédents concernant la population adolescente $[20,57]$ de même que

Tableau 3 Synthèses des résultats à l'analyse de régression multiple avec comme variables explicatives des conduites à risques : l'âge, les dimensions du tempérament, et le niveau ce conscience émotionnelle.

\begin{tabular}{|c|c|c|c|c|c|}
\hline \multirow[t]{2}{*}{ Modèle } & \multicolumn{5}{|c|}{ Variable à expliquer } \\
\hline & 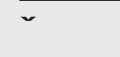 & $t$ & $R^{2}$ & $F$ & $p$ \\
\hline Conduites à Risques & & & 0,33 & 30,78 & $<0,0001$ \\
\hline Sexe & $-0,017$ & $-0,429$ & & & 0,668 \\
\hline Âge & 0,057 & 1,429 & & & 0,154 \\
\hline Recherche de nouveauté & 0,315 & 7,078 & & & $<0,0001$ \\
\hline Évitement du danger & $-0,011$ & $-0,267$ & & & 0,789 \\
\hline Persistance & $-0,027$ & $-0,682$ & & & 0,496 \\
\hline Dépendance à la récompense & 0,070 & 1,860 & & & 0,060 \\
\hline NCE Sujet & $-0,238$ & $-4,690$ & & & $<0,0001$ \\
\hline NCE Autre personne & $-0,186$ & $-3,866$ & & & 0,0001 \\
\hline NCE Sujet & & & 0,12 & 16,71 & $<0,0001$ \\
\hline Recherche de nouveauté & $-0,199$ & $-3,990$ & & & $<0,0001$ \\
\hline Évitement du danger & 0,194 & 4,069 & & & $<0,0001$ \\
\hline Persistance & $-0,054$ & $-1,257$ & & & 0,209 \\
\hline Dépendance à la récompense & 0,018 & 0,403 & & & 0,687 \\
\hline NCE Autre personne & & & 0,02 & 3,02 & 0,02 \\
\hline Recherche de nouveauté & $-0,057$ & $-1,080$ & & & 0,281 \\
\hline Évitement du danger & 0,126 & 2,497 & & & 0,013 \\
\hline Persistance & $-0,021$ & $-0,468$ & & & 0,640 \\
\hline Dépendance à la récompense & $-0,050$ & $-1,032$ & & & 0,302 \\
\hline
\end{tabular}


ceux ayant évalué la dimension proche de la recherche de sensations $[40,44]$ comme facteurs explicatifs de la prise de risque. Plus avant, nous remarquons que le niveau de conscience émotionnelle joue un rôle important dans la prise de risque. Ainsi, plus les sujets auront un niveau de conscience émotionnelle faible, en particulier dans sa dimension soi, associé à une forte recherche de nouveauté, plus ils auront tendance à s'engager dans des conduites à risques. Ce résultat vient soutenir ceux issus d'une précédente recherche réalisée sur un échantillon plus modeste [4], ainsi que les observations cliniques mettant en relief les difficultés des adolescents "preneurs de risques» à rendre compte de leurs éprouvés subjectifs.

Ces résultats appellent à plusieurs réflexions concernant les limites de cette étude. Premièrement, les outils utilisés n'ont pas fait l'objet d'une revalidation proprement dite auprès d'adolescents, mais d'une adaptation, décrite plus haut. Néanmoins, leur utilisation dans des études antérieures a montré leur intérêt [41,42]. Deuxièmement, l'évaluation a été pour l'essentiel réalisée par autoquestionnaires, à l'exception des conduites à risques, ce qui peut amener à biaiser certaines réponses par désirabilité sociale. Cependant, comme nous l'avons déjà évoqué, nous avons réalisé une double évaluation de ces conduites, à travers l'hétéroévaluation par un adulte du cadre scolaire; par ailleurs, l'échelle d'évaluation du niveau de conscience émotionnelle possède une structure spécifique et une cotation par l'examinateur qui permet de limiter ce biais, comme le montre l'absence de corrélation avec l'échelle de désirabilité sociale de Marlowe-Crowne [28]. En revanche, on ne peut rejeter l'hypothèse que des sujets aient estimé peu important de décrire l'entière complexité de leurs éprouvés subjectifs [6]. Troisièmement, la structure de cette étude étant transversale et non longitudinale, elle ne permet pas de dégager une relation de causalité linéaire entre les variables. L'hypothèse d'un sens de la relation allant des variables psychologiques vers les comportements est soutenue par des travaux existants mais devra être confirmée par des études expérimentales et longitudinales. Enfin, la dimension culturelle dans l'expression des émotions ainsi que le niveau intellectuel des sujets n'ont pas été contrôlés du fait de l'absence de données sociodémographiques; une investigation ultérieure pourra être mené en tenant compte des liens entre capacités cognitives et fonctionnement émotionnel [27].

À l'issue de ce travail, plusieurs propositions de compréhension de nos résultats peuvent être formulées. Une première interprétation pourrait reposer sur une articulation entre les dimensions émotionnelles, le comportement produit et sa répétition, en analogie avec certains modèles des addictions. En effet, nous observons que l'engagement dans des conduites à risques semble lié à une forte recherche de nouveauté associée à une faible conscience émotionnelle. Cette tendance à rechercher les sensations et les stimulations pourrait être comprise comme un moyen de lutter contre un vécu de «vide interne» associé à une vie imaginaire pauvre. Une seconde possibilité de lire ces résultats serait que les adolescents remplaceraient les émotions (et éprouvés) - internes et intolérables-par des sensations - externes et contrôlables donc supportables.

Enfin, une interprétation alternative pourrait considérer la recherche de stimulations répétées comme une tentative, certes infructueuse, de transformation de l'expérience sensorielle en expérience subjective, par l'incapacité à entrer dans un processus où l'évaluation cognitive pourrait jouer son rôle de traitement de l'information amenant à l'expérience consciente de l'émotion. Si l'on se réfère aux modèles tempéramentaux la recherche de nouveauté est indispensable au petit enfant pour explorer son environnement. Le sujet développe ensuite la capacité à réguler ses comportements, en inhibant certains et en différant d'autres. Dans ce processus, la capacité à disposer d'une information émotionnelle permettant l'orientation et l'adaptation des comportements est essentielle. En son absence, des difficultés ultérieures pourraient ainsi survenir dans la capacité à: réguler ses comportements faute d'information émotionnelle appropriée; se construire des représentations psychiques émotionnelles complexes permettant des interactions adaptées à l'environnement social.

Ainsi, le fait de ressentir les émotions à un niveau plus différencié permettrait de passer de l'intensité de l'éprouvé subjectif au traitement cognitif et à l'adaptation comportementale [50]. Des indices de ce fonctionnement ont pu être retrouvés dans une étude exploratoire [5]. Au regard de l'histoire de nombreux adolescents en grande difficulté vus en consultation et engagés dans des prises de risques répétées, nous pensons qu'une faible capacité à mentaliser les états émotionnels est la conséquence d'une vulnérabilité interne majeure aux éprouvés subjectifs. Cette vulnérabilité pourrait trouver son origine dans des arrêts développementaux précoces liés à des traumas infantiles.

\section{Conclusion}

Les relations entre émotions et tempérament dans les conduites à risques sont complexes, et demandent encore à être précisées. En effet, si nous avons pu mettre ici en évidence des dimensions pouvant être considérées dans leur articulation comme des facteurs de vulnérabilité, l'une des limites de notre étude concerne l'aspect subjectif de l'expérience émotionnelle qui n'a pas été évalué. Néanmoins, elle constitue une avancée significative soutenant une nécessaire prise en compte des troubles de la construction subjective de l'expérience émotionnelle, ainsi que de ses déficits dans les prises en charge d'adolescents présentant des conduites à risques. Ainsi, l'utilisation de techniques psychothérapiques impliquant les dimensions attentionnelles et évaluatives de l'expérience émotionnelle pourrait permettre de transformer la recherche de stimulations répétitives en ressources adaptatives. Dans ce cadre, l'utilisation de médiateurs tels que certaines activités sportives impliquant la dimension de l'expérience du corps (plongée sous-marine par exemple) associés à des temps d'élaboration de l'expérience émotionnelle vécue pourraient utilement être explorés.

\section{Déclaration d'intérêts}

Les auteurs déclarent ne pas avoir de conflits d'intérêts en relation avec cet article. 


\section{Remerciements}

Nous tenons à exprimer notre gratitude au Dr Sylvie Berthoz qui nous a transmis la LEAS ainsi que les instructions concernant son utilisation.

\section{Références}

[1] Ball SA, Tennen H, Poling JC, et al. Personality, temperament, and character dimensions and the DSM-IV personality disorders in substance abusers. J Abnorm Psychol 1997;106:545-53.

[2] Bochand L, Nandrino JL. Niveaux de conscience émotionnelle chez les sujets alcoolodépendants et abstinents. Encéphale 2010;36:334-9.

[3] Bonnet A, Pedinielli JL, Romain F, et al. Bien-être subjectif et régulation émotionelle dans les conduites à risque. Cas de la plongée sous-marine. Encéphale 2003;29:488-97.

[4] Bréjard V, Bonnet A, Pedinielli JL. Développement cognitivoémotionnel, régulation des émotions et comportements à risques: une étude exploratoire chez l'adolescent. Neuropsychiatr Enfance Adolesc 2005;53:395-400.

[5] Brejard V, Pedinielli JL, Rouan G. Hypothèse d'un dysfonctionnement émotionnel chez des adolescents présentant des comportements à risque: une étude exploratoire. Encéphale 2006;32:413-20.

[6] Bydlowski S, Corcos M, Jeammet P, et al. Emotion-processing deficits in eating disorders. Int J Eat Disord 2005;37:321-9.

[7] Bydlowski S, Corcos M, Paterniti S, et al. Validation de la version francaise de l'échelle des niveaux de conscience émotionnelle. Encéphale 2002;28:310-20.

[8] Carton S. La recherche de sensations: quel traitement de l'émotion? Psychotropes 2005;11:121-44.

[9] Cloninger CR, Sigvardsson S, Przybeck TR, et al. A systematic method for clinical description and classification of personality variants. A proposal. Arch Gen Psychiatry 1987;44:573-88.

[10] Cloninger CR, Sigvardsson S, Bohman M. Childhood personality predicts alcohol abuse in young adults. Alcohol Clin Exp Res 1988;12:494-505.

[11] Cloninger CR, Sigvardsson S, Gilligan SB, et al. Genetic heterogeneity and the classification of alcoholism. Adv Alcohol Subst Abuse 1988;7:3-16.

[12] Cloninger CR, Sigvardsson S, Przybeck TR, et al. Personality antecedents of alcoholism in a national area probability sample. Eur Arch Psychiatry Clin Neurosci 1995;245:239-44.

[13] Cloninger CR, Svrakic DM, Przybeck TR. A psychobiological model of temperament and character. Arch Gen Psychiatry 1993;50:975-90.

[14] Cohen JD. Statistical power analysis for the behavioral sciences. Hillsdale, NJ: Erlbaum; 1988.

[15] Desrichard O, Denarie V. Sensation seeking and negative affectivity as predictors of risky behaviors: a distinction between occasional versus frequent risk-taking. Addict Behav 2005;30:1449-53.

[16] Donges US, Kersting A, Dannlowski U, et al. Reduced awareness of others' emotions in unipolar depressed patients. J Nerv Ment Dis 2005;193:331-7.

[17] Gilboa-Schechtman E, Avnon L, Zubery E, et al. Emotional processing in eating disorders: specific impairment or general distress related deficiency? Depress Anxiety 2006;23:331-9.

[18] Grunbaum JA, Kann L, Kinchen S, et al. Youth risk behavior surveillance-United States, 2003 (Abridged). J Sch Health 2004;74:307-24.

[19] Guilbaud O, Loas G, Corcos M, et al. L'alexithymie dans les conduites de dépendance et chez le sujet sa valeur en population française et francophone: alexithymia in addictive behaviors in healthy subjects: results of a study in French speaking subjects. Ann Med Psychol 2002;160:77-85.

[20] Hale RL, Whiteman S, Muehl K, et al. Tridimensional personality traits of college student marijuana users. Psychological Rep 2003;92:661-6.

[21] Hessler DM, Katz LF. Brief report: associations between emotional competence and adolescent risky behavior. J Adolescence 2010;33:241-6.

[22] Johnson TJ, Cropsey KL. Sensation seeking and drinking game participation in heavy-drinking college students. Addictive Behaviors 2000;25:109-16.

[23] Jouanne C, Edel Y, Carton S. Déficits émotionnels chez des patients polytoxicomanes. Ann Med Psychol 2005;163: 625-30.

[24] Kalichman SC, Tannenbaum L, Nachimson D. Personality and cognitive factors influencing substance use and sexual risk for HIV infection among gay and bisexual men. Psychology Addict Behav 1998;12:262-71.

[25] Kelley AE, Schochet T, Landry CF. Risk taking and novelty seeking in adolescence: introduction to part I. Ann N Y Acad Sci 2004;1021:27-32.

[26] Kojima M, Furukawa T, Nagaya T, et al. Smoking, depression and alexithymia. J Psychosom Res 2003;55:156.

[27] Kopp C. Emotional distress and control in young children. In: Eisenberg N, Fabes RA, editors. Emotion and its regulation in early development. New directions in child development. San Francisco: Jossey-Bass; 1992. p. 41-56.

[28] Lane RD, Quinlan DM, Schwartz GE, et al. The Levels of Emotional Awareness Scale: a cognitive-developmental measure of emotion. J Pers Assess 1990;55:124-34.

[29] Lane RD, Schwartz GE. Levels of emotional awareness: a cognitive-developmental theory and its application to psychopathology. Am J Psychiatry 1990;144:133-43.

[30] Lane RD, Schwartz GE. The neuropsychophysiology of emotion. Funct Neurol 1990;5:263-6.

[31] Lane RD, Sechrest L, Riedel R, et al. Pervasive emotion recognition deficit common to alexithymia and the repressive coping style. Psychosom Med 2000;62:492-501.

[32] Le Bon O, Staner L, Tecco J. Questionnaire de personnalité tridimensionnel (TPQ) : validation chez une population contrôle francophone. Encéphale 1998;24:40-5.

[33] Lépine JP, Pelissolo A, Téodorescu R, et al. Évaluation des propriétés psychométriques de la version française du questionnaire tridimensionnel de la personnalité (TPQ). Encéphale 1994;20:747-53.

[34] Liappas J, Peppas E, Rabavilas AD, et al. The relationship between alexithymia and depression-like symptomatology in drue users. Eur Neuropsychopharmacol 1998;8:5-14.

[35] Loas G, Otmani O, Lecercle $C$, et al. Relationships between the emotional and cognitive components of alexithymia and dependency in alcoholics. Psychiatr Res 2000;96: 63-74.

[36] Lumley MA, Roby KJ. Alexithymia and pathological gambling. Psychother Psychosom 1995;63:201-6.

[37] Masse LC, Tremblay RE. Behavior of boys in kindergarten and the onset of substance use during adolescence. Arch Gen Psychiatry 1997;54:62-8.

[38] Michel G. Recherche de sensation et de nouveauté à l'adolescence: trait développmental, état ou facteur de vulnérabilité aux conduites de consommation et à risques. Paris: Université Paris VII; 1999.

[39] Michel G, Carton S, Jouvent R. Recherche de sensations et anhedonie dans les conduites de prise de risque. Étude d'une population de sauteurs à l'élastique (Benji). Encéphale 1997;23:403-11.

[40] Michel G, Le Heuzey MF, Purper-Ouakil D, et al. Recherche de sensations et conduites à risque chez l'adolescent. Ann Med Psychol 2001;159:708-16. 
[41] Michel G, Mouren-Simeoni M-C, Perez-Diaz F. The Tridimensional Personality Questionnaire and the Sensation-Seeking scale in depressed adolescent: state versus trait issues. Eur Child Adolesc Psychiatry 1999;8:126.

[42] Michel G, Mouren-Simeoni MC, Purper-Ouakil D. Measurement of personality in adolescence: a longiutudinal study off ten sensation seeking scale (SSS) and the tridimensional personality questionnaire (TPQ). Eur Child Adolesc Psychiatry 1999;8:135.

[43] Michel G, Purper-Ouakil D, Mouren-Simeoni MC. Clinique et recherche sur les conduites à risques chez l'adolescent. Neuropsychiatr Enfance Adolesc 2006;54:62-76.

[44] Michel G, Purper-Ouakil D, Mouren-Siméoni MC. Facteurs de risques des conduites de consommation de substances psychoactives à l'adolescence. Ann Med Psychol 2001;159:622-31.

[45] Novick-Kline P, Turk CL, Mennin DS, et al. Level of emotional awareness as a differentiating variable between individuals with and without generalized anxiety disorder. J Anxiety Disord 2005; 19:557-72.

[46] Parker J, Wood LM, Bond BJ, et al. Alexithymia in young adulthood: a risk factor for pathological gambling. Psychother Psychosom 2005;74:51-5.

[47] Plomin R. Childhood temperament. In: Lahey B, Kazdin A, editors. Advances in clinical child psychology. New york: Plenum; 1983.

[48] Rieffe C, Oosterveld P, Miers AC. Emotion awareness anc internalising symptoms in children and adolescents: the Emotion Awareness Questionnaire revised. Pers Ind Differ 2008;45:756-61.

[49] Rouan G, Pedinielli JL. Émotion et psychopathologie cognitive. In: Channouf H, Rouan G, editors. Émotions et cognitions. Bruxelles: De Boeck; 2000. p. 225-55.
[50] Scherer KR, Sangsue J. Le système mental en tant que composante de l'émotion. In: Kirouac $G$, editor. Cognitions et émotions. Coimbra: Presses Universitaires de Coimbra; 2004. p. 11-36.

[51] Speranza M, Corcos M, Stéphan P. Alexithymia, depressive experiences, and dependency in addictive disorders. Subst Use Misuse 2004;39:551-79.

[52] Sultan S, Pipon A. Une application des modèles dimensionnels à la sexualité à risque: personnalité et usage du préservatif. Rev Eur Psychol Appl 1996;46:145-53.

[53] Volkow ND, Wang GJ, Fowler JS, et al. Decreases in dopamine receptors but not in dopamine transporters in alcoholics. Alcohol Clin Exp Res 1996;20:1594-8.

[54] Vukov M, Baba-Milkic N, Lecic D, et al. Personality dimensions of opiate addicts. Acta Psychiatr Scand 1995;91: $103-7$.

[55] Wills TA, Sandy JM, Shinar O. Cloninger's constructs related to substance use level and problems in late adolescence: a mediational model based on self-control and coping motives. Exp Clin Psychopharmacol 1999;7:122-34.

[56] Wills TA, Vaccaro D, McNamara G. Novelty seeking, risk taking, and related constructs as predictors of adolescent substance use: an application of Cloninger's theory. J Subst Abuse 1994;6:1-20.

[57] Wills TA, Windle M, Cleary SD. Temperament and novelty seeking in adolescent substance use: convergence of dimensions of temperament with constructs from Cloninger's theory. J Pers Soc Psychol 1998;74:387-406.

[58] Zuckerman M, Cloninger CR. Relationships between Cloninger's, Zuckerman's, and Eyesenck's dimensions of personality. Pers Ind Differ 1996;21:283-5. 